\title{
Necessary Optimality Conditions for Multi-Objective Semi-Infinite Variational Problem
}

\author{
Bharti Sharma1, Promila Kumar² \\ ${ }^{1}$ Department of Mathematics, University of Delhi, New Delhi, India \\ ${ }^{2}$ Department of Mathematics, Gargi College, University of Delhi, New Delhi, India \\ Email: bharti.sharma3135@yahoo.in, kumar.promila@gmail.com
}

Received 30 November 2015; accepted 18 January 2015; published 22 January 2016

Copyright (C) 2016 by authors and Scientific Research Publishing Inc.

This work is licensed under the Creative Commons Attribution International License (CC BY). http://creativecommons.org/licenses/by/4.0/

(c) () Open Access

\begin{abstract}
In this paper, necessary optimality conditions for a class of Semi-infinite Variational Problems are established which are further generalized to a class of Multi-objective Semi-Infinite Variational Problems. These conditions are responsible for the development of duality theory which is an extremely important feature for any class of problems, but the literature available so far lacks these necessary optimality conditions for the stated problem. A lemma is also proved to find the topological dual of $\left(L^{2}(I, \mathbb{R})\right)^{\mathbb{N}}$ as it is required to prove the desired result.
\end{abstract}

\section{Keywords}

Semi-Infinite, Variational Problem, Efficient Solution, Necessary Optimality Conditions

\section{Introduction}

A Semi-infinite Programming Problem (SIP) [1]-[3] is an optimization problem in which the index set of inequality constraints is an arbitrary and not necessarily finite set. It has wide variety of applications in various fields like economics, engineering, mathematical physics and robotics. While browsing the literature, we observe that much attention has been paid to SIP which is static in nature in the sense that time does not enter into consideration. Whereas in practical problems we come across situations where time plays an important role and hence cannot be neglected.

Semi-infinite Programming Problem is tightly interwoven with Variational Problem [4]-[9]. Both these subjects have undergone independent development, hence mutual adaptation of ideas and techniques have always been appreciated. 
In this article, we propose Semi-infinite Variational Problem for which necessary optimality conditions are established. These optimality conditions are further extended to Multi-objective Semi-infinite Variational Problem (MSVP). We also clarify, with proper reasoning, certain points which were left for later validation in [9].

Necessary optimality conditions are important because these conditions lay down foundation for many computational techniques in optimization problems as they indicate when a feasible point is not optimal. At the same time these conditions are useful in the development of numerical algorithms for solving certain optimization problems. Further, these conditions are also responsible for the development of duality theory on which there exists an extensive literature and a substantial use of which (duality theory) has been made in theoretical as well as computational applications in many diverse fields. While browsing the literature, we found that necessary optimality conditions were not proved for the class of semi-infinite variational problems.

The paper is organized as follows: In section 2 some basic definitions and preliminaries are given. Section 3 deals with necessary optimality conditions for semi-infinite variational problem; single objective as well as multiobjective. In section 4 , we prove a lemma which is required to prove necessary optimality conditions of section 3 , for semi-infinite variational problem.

\section{Definitions and Preliminaries}

Let $E$ be a topological vector space over the field of real numbers and $E^{\prime}$ denotes the topological dual space of $E$. For a set $C \subset E$, the topological polar cone $C^{\prime}$ of $C$ is $C^{\prime}=\left\{\xi \in E^{\prime} \mid \xi(c) \geq 0\right.$, for all $\left.c \in C\right\}$. Let $r$ and $n$ be two positive integers. For a given real interval $I=[a, b]$, let $x: I \rightarrow \mathbb{R}^{n}$ be a piecewise smooth state function with its derivative $\dot{x}$. For notational convenience we write $x, \dot{x}$ in place of $x(t), \dot{x}(t)$. Let $M=\{1,2, \cdots, r\}$, $f^{i}: I \times \mathbb{R}^{n} \times \mathbb{R}^{n} \rightarrow \mathbb{R}, i \in M$ and $g^{j}: I \times \mathbb{R}^{n} \times \mathbb{R}^{n} \rightarrow \mathbb{R}, j \in \mathbb{N}$ be continuously differentiable functions with respect to each of their argument. We also denote the partial derivative of $f^{i}, i \in M$ with respect to $t, x$ and $\dot{x}$ by $f_{t}^{i}, f_{x}^{i}, f_{\dot{x}}^{i}$ respectively. Analogously, we write the partial derivative of $g^{i}, i \in \mathbb{N}$. For the sake of notational convenience we write $f_{x}^{j}(t)$ for $f_{x}^{j}(t, x(t), \dot{x}(t))_{\mathrm{T}}$ and $f_{\dot{x}}^{j}(t)$ for $f_{\dot{x}}^{j}(t, x(t), \dot{x}(t))$ for $j=1,2, \cdots, r$.

For any $x=\left(x^{1}, x^{2}, \cdots, x^{n}\right)^{\mathrm{T}}, y=\left(y^{1}, y^{2}, \cdots, y^{n}\right)^{\mathrm{T}}$ in n-dimensional Euclidean space $\mathbb{R}^{n}$,

1) $x=y \Leftrightarrow x^{i}=y^{i}$ for all $i=1,2, \cdots, n$.

2) $x<y \Leftrightarrow x^{i}<y^{i}$ for all $i=1,2, \cdots, n$.

3) $x \leqq y \Leftrightarrow x^{i} \leq y^{i}$ for all $i=1,2, \cdots, n$.

4) $x \leq y \Leftrightarrow x \leqq y$ and $x \neq y$.

Let $\mathbb{R}_{+}^{n}$ and int $\mathbb{R}_{+}^{n}$ denote the non negative and positive orthant of $\mathbb{R}^{n}$ respectively. Let $X$ be the space of piecewise smooth state functions $x: I \rightarrow \mathbb{R}^{n}$ which equipped with the norm $\|x\|=\|x\|_{\infty}+\|D x\|_{\infty}$, where the differential operator $D$ is given by

$u=D x \Leftrightarrow x(t)=x(a)+\int_{a}^{t} u(s)$ ds. Therefore, $D=\frac{\mathrm{d}}{\mathrm{d} t}$ except at discontinuities.

Consider the following Multi-objective Semi-infinite Variational Problem (MSVP):

$$
\text { (MSVP) Minimize }\left(\int_{a}^{b} f^{1}(t, x, \dot{x}) \mathrm{d} t, \cdots, \int_{a}^{b} f^{r}(t, x, \dot{x}) \mathrm{d} t\right)
$$

subject to

$$
\begin{aligned}
& g^{i}(t, x, \dot{x}) \leqq 0, i \in \mathbb{N}, t \in I, x \in X, \\
& x(a)=0, x(b)=0 .
\end{aligned}
$$

$\bar{X}=\{x \in X \mid x(a)=0, x(b)=0\}$ with the norm defined as above is a Banach space.

Let $X_{0}=\left\{x \in X \mid g^{i}(t, x, \dot{x}) \leqq 0, i \in \mathbb{N}, t \in I, x(a)=0, x(b)=0\right\}$ be the set of all feasible solutions of (MSVP).

Definition 1 A point $\bar{x} \in X_{0}$ is said to be an efficient solution for (MSVP) if there is no other $x \in X_{0}$ such that

$$
\begin{aligned}
& \int_{a}^{b} f^{i}(t, x, \dot{x}) \mathrm{d} t \leq \int_{a}^{b} f^{i}(t, \bar{x}, \dot{\bar{x}}) \mathrm{d} t, \quad \text { for all } i \in M \text { and, } \\
& \int_{a}^{b} f^{j}(t, x, \dot{x}) \mathrm{d} t<\int_{a}^{b} f^{j}(t, \bar{x}, \dot{\bar{x}}) \mathrm{d} t, \quad \text { for at least one } j \in M
\end{aligned}
$$




\section{Necessary Optimality Conditions}

Let us first prove necessary optimality conditions for the following single objective Semi-infinite Variational Problem (SVP):

$$
\text { (SVP) Minimize } \int_{a}^{b} \phi(t, x, \dot{x}) \mathrm{d} t
$$

subject to

$$
\begin{aligned}
& g^{i}(t, x, \dot{x}) \leqq 0, i \in \mathbb{N}, t \in I, x \in X, \\
& x(a)=0, x(b)=0 .
\end{aligned}
$$

where $\phi: I \times \mathbb{R}^{n} \times \mathbb{R}^{n} \rightarrow \mathbb{R}$ is continuously differentiable function with respect to each of its argument.

The problem (SVP) may be rewritten as Cone Constrained Problem (CCP):

$$
\text { (CCP) Minimize } \Phi(x)
$$

subject to

$$
-G(x) \in K, x \in \bar{X}
$$

where $\Phi: \bar{X} \rightarrow \mathbb{R}$ is defined as

$$
\begin{gathered}
\Phi(x)=\int_{a}^{b}\{\phi(t, x, \dot{x})\} \mathrm{d} t, x \in \bar{X} \\
L^{2}(I, \mathbb{R})=\left\{f: I \rightarrow \mathbb{R} \text { such that } f \text { is measurable and } \int_{a}^{b}|f(t)|^{2} \mathrm{~d} \mu(t)<\infty\right\} \\
=\left\{f: I \rightarrow \mathbb{R} \text { such that } f \text { is measurable and } \int_{a}^{b}|f(t)|^{2} \mathrm{~d} t<\infty\right\},
\end{gathered}
$$

where $\mu$ is Lebesgue measure.

$$
\begin{aligned}
& \left(L^{2}(I, \mathbb{R})\right)^{\mathbb{N}}=\left\{f=\left(f^{i}\right)_{i \in \mathbb{N}} \mid f^{i} \in L^{2}(I, \mathbb{R}), i \in \mathbb{N}\right\}, \\
& K=\left\{\left(\gamma^{i}\right)_{i \in \mathbb{N}}=\gamma \in\left(L^{2}(I, \mathbb{R})\right)^{\mathbb{N}} \mid \gamma^{i}(t) \geqq 0, i \in \mathbb{N}, t \in I\right\} ;
\end{aligned}
$$

$G: \bar{X} \rightarrow\left(L^{2}(I, \mathbb{R})\right)^{\mathbb{N}}$ is defined as

$$
(G(x))^{i}(t)=g^{i}(t, x, \dot{x}), i \in \mathbb{N}, t \in I, x \in \bar{X}
$$

Theorem 2 Let $\bar{X}$ be an optimal solution of (SVP). Then there exist $\bar{\tau} \in \mathbb{R}_{+}$and piecewise smooth functions $\bar{\lambda}^{i}: I \rightarrow \mathbb{R}, \bar{\lambda}^{i} \neq 0$ for finitely many $i \in \mathbb{N}$ such that

$$
\begin{aligned}
& \bar{\tau} \phi_{\bar{x}}(t)+\sum_{i \in \mathbb{N}} \bar{\lambda}^{i}(t) g_{\bar{x}}^{i}(t)=\frac{\mathrm{d}}{\mathrm{d} t}\left[\bar{\tau} \phi_{\overline{\bar{x}}}(t)+\sum_{i \in \mathbb{N}} \bar{\lambda}^{i}(t) g_{\overline{\bar{x}}}^{i}(t)\right], t \in I, \\
& \int_{a}^{b} \sum_{i \in \mathbb{N}} \bar{\lambda}^{i}(t) g^{i}(t, \bar{x}, \dot{\bar{x}}) \mathrm{d} t=0, \\
& \left(\tau, \bar{\lambda}=\left(\bar{\lambda}^{i}\right)_{i \in \mathbb{N}}\right) \neq 0, \bar{\lambda}^{i}(t) \geqq 0 \quad \text { a.e., } t \in I, i \in \mathbb{N} .
\end{aligned}
$$

Proof. Since $\bar{x}$ is an optimal solution of (SVP), so is of (CCP). Therefore there exist $\bar{\tau} \in \mathbb{R}_{+}$and $\bar{y} \in K^{\prime}$ (topological polar cone of $K$ ) [10] such that

$$
\begin{aligned}
& \bar{\tau} \Phi^{\prime}(\bar{x})+\bar{y} G^{\prime}(\bar{x})=0, \\
& \bar{y} G(\bar{x})=0 .
\end{aligned}
$$


where $\Phi^{\prime}(\bar{x})$ and $G^{\prime}(\bar{x})$ are Frechet derivatives of $\Phi$ and $G$ at $\bar{x}$.

Also for every $v \in \bar{X}$,

$$
\begin{aligned}
& (\Phi)^{\prime}(\bar{x})(v)=\int_{a}^{b}\left\{\phi_{\bar{x}}(t) v(t)+\phi_{\bar{x}}(t) \dot{v}(t)\right\} \mathrm{d} t \\
& \left(G^{\prime}(\bar{x})\right)^{i}(v)(t)=g_{\bar{x}}^{i}(t) v(t)+g_{\overline{\bar{x}}}^{i}(t) \dot{v}(t), i \in \mathbb{N}, t \in I .
\end{aligned}
$$

Since $\bar{y} \in K^{\prime}$,

$$
\Rightarrow \bar{y} \in\left(\left(L^{2}(I, \mathbb{R})\right)^{\mathbb{N}}\right)^{\prime} .
$$

By Lemma 1 (proved in Section 4)

$$
\bar{y}=\left(\bar{y}^{i}\right)_{i \in \mathbb{N}}, \bar{y}^{i} \in L^{2}(I, \mathbb{R}), \bar{y}^{i} \neq 0 \text {, for finitely many } i \in \mathbb{N} .
$$

Let $S=\left\{i \in \mathbb{N} \mid \bar{y}^{i} \neq 0\right\}$. For $i \in S, \bar{y}^{i} \in L^{2}(I, \mathbb{R})$, by Riesz representation theorem [11] there exist $\bar{\lambda}^{i} \in L^{2}(I, \mathbb{R})$ such that

$$
\bar{y}^{i}(h)=\int_{a}^{b} \bar{\lambda}^{i}(t) h(t) \mathrm{d} t, \text { for all } h \in L^{2}(I, \mathbb{R}),
$$

for $i \notin S$, choose $\bar{\lambda}^{i}=0$, therefore for any $v \in\left(L^{2}(I, \mathbb{R})\right)^{\mathbb{N}}$,

$$
\bar{y}(v)=\int_{a}^{b} \sum_{i \in \mathbb{N}} \bar{\lambda}^{i}(t) v^{i}(t) \mathrm{d} t .
$$

Substituting $v=G(\bar{x})$ in (16) and using (12), we arrive at (9).

Now it follows from (11)

$$
\bar{\tau} \Phi^{\prime}(\bar{x})(h)+\bar{y} G^{\prime}(\bar{x})(h)=0, \text { for all } h \in \bar{X} .
$$

(13) along with (16) implies

$$
\int_{a}^{b}\left[\bar{\tau} \phi_{\bar{x}}(t) h(t)+\bar{\tau} \phi_{\dot{\bar{x}}}(t) \dot{h}(t)+\sum_{i \in \mathbb{N}} \bar{\lambda}^{i}(t) G^{\prime}(\bar{x})^{i}(h)(t)\right] \mathrm{d} t=0 \text {, for all } h \in \bar{X} .
$$

On using (14), we get

$$
\begin{aligned}
& \int_{a}^{b}\left[\bar{\tau} \phi_{\bar{x}}(t) h(t)+\bar{\tau} \phi_{\bar{x}}(t) \dot{h}(t)+\sum_{i \in \mathbb{N}} \bar{\lambda}^{i}(t) g_{\bar{x}}^{i}(t) h(t)+\sum_{i \in \mathbb{N}} \bar{\lambda}^{i}(t) g_{\dot{\bar{x}}}^{i}(t) \dot{h}(t)\right] \mathrm{d} t=0, \text { for all } h \in \bar{X} \\
& \Rightarrow \int_{a}^{b}\left[\left(\bar{\tau} \phi_{\bar{x}}(t)+\sum_{i \in \mathbb{N}} \bar{\lambda}^{i}(t) g_{\bar{x}}^{i}(t)\right) h(t)+\left(\bar{\tau} \phi_{\overline{\bar{x}}}(t)+\sum_{i \in \mathbb{N}} \bar{\lambda}^{i}(t) g_{\overline{\bar{x}}}^{i}(t)\right) \dot{h}(t)\right] \mathrm{d} t=0, \text { for all } h \in \bar{X}
\end{aligned}
$$

Integrating by parts the following function and using boundary condition of $h$,

$$
\int_{a}^{b}\left[\left(\bar{\tau} \phi_{\overline{\bar{x}}}(t)+\sum_{i \in \mathbb{N}} \bar{\lambda}^{i}(t) g_{\dot{\bar{x}}}^{i}(t)\right) \dot{h}(t)\right] \mathrm{d} t=\int_{a}^{b}-\frac{\mathrm{d}}{\mathrm{d} t}\left(\bar{\tau} \phi_{\overline{\bar{x}}}(t)+\sum_{i \in \mathbb{N}} \bar{y}^{i}(t) g_{\dot{\bar{x}}}^{i}(t)\right) h(t) \mathrm{d} t .
$$

Using above equation in (20), we get

$$
\int_{a}^{b}\left[\left(\bar{\tau} \phi_{\bar{x}}(t)+\sum_{i \in \mathbb{N}} \bar{\lambda}^{i}(t) g_{\bar{x}}^{i}(t)\right)-\frac{\mathrm{d}}{\mathrm{d} t}\left(\bar{\tau} \phi_{\overline{\bar{x}}}(t)+\sum_{i \in \mathbb{N}} \bar{y}^{i}(t) g_{\overline{\bar{x}}}^{i}(t)\right)\right] h(t) \mathrm{d} t=0 \text {, for all } h \in \bar{X}
$$

By fundamental theorem of calculus of variation [12]

$$
\left(\bar{\tau} \phi_{\bar{x}}(t)+\sum_{i \in \mathbb{N}} \bar{\lambda}^{i}(t) g_{\bar{x}}^{i}(t)\right)=\frac{\mathrm{d}}{\mathrm{d} t}\left(\bar{\tau} \phi_{\overline{\bar{x}}}(t)+\sum_{i \in \mathbb{N}} \bar{y}^{i}(t) g_{\bar{x}}^{i}(t)\right), t \in I .
$$


Claim 1: $\bar{\lambda}^{i}(t) \geq 0$ a.e., $t \in I, i \in S$.

Without loss of generality assume that $S=\{1,2, \cdots, s\}$.

Since $\bar{y} \in K^{\prime}$,

$$
\begin{aligned}
& \Rightarrow \bar{y}(y) \geq 0, \text { for all } y \in K, \\
& \Rightarrow \int_{a}^{b} \sum_{i \in S} \bar{\lambda}^{i}(t) y^{i}(t) \mathrm{d} t \geq 0, \text { for all } y^{i} \in L^{2}(I, \mathbb{R}) \text { such that } y^{i}(t) \geq 0, t \in I, i \in S .
\end{aligned}
$$

In particular

$$
\int_{a}^{b} \bar{\lambda}^{1}(t) y^{1}(t) \mathrm{d} t \geq 0 \text {, for all } y^{1} \in L^{2}(I, \mathbb{R}) \text { such that } y^{1}(t) \geq 0, t \in I .
$$

Claim 2: $\mu(A)=0$, where $A=\left\{t \in I \mid \bar{\lambda}^{1}(t)<0\right\}$.

Let if possible $\mu(A)>0$, then $\int_{A} \bar{\lambda}^{1}(t) \mathrm{d} t<0$.

Define $\hat{y}^{1}(t)= \begin{cases}1 & t \in A \\ 0 & t \notin A .\end{cases}$

Then $\hat{y}^{1}(t) \geq 0, t \in I$, but $\int_{a}^{b} \bar{\lambda}^{1}(t) \hat{y}^{1}(t) \mathrm{d} t=\int_{A} \bar{\lambda}^{1}(t) \mathrm{d} t<0$, a contradiction.

Hence Claim 2 holds, that is, $\bar{\lambda}^{1}(t) \geq 0$ a.e., $t \in I$.

Using the same argument $\bar{\lambda}^{i}(t) \geq 0$ a.e., $t \in I, i=2, \cdots, s$. Hence claim 1 also holds.

The relations (16) are generally valid only if $\bar{\lambda}(\cdot)=\left(\bar{\lambda}^{i}(\cdot)\right)_{i \in \mathbb{N}}$ is Schwarz distribution. Condition (8) is a linear first order differential equation for $\bar{\lambda}(\cdot)$, therefore for given $\bar{x}$, equation (8) is solvable for piecewise smooth function $\bar{\lambda}(\cdot)$ [9] [13].

Theorem 3 (Necessary Optimality Conditions) Let $\bar{x}$ be a normal efficient solution for (MSVP). Then there exist $\left(\bar{\tau}^{1}, \bar{\tau}^{2}, \cdots, \bar{\tau}^{r}\right) \in \mathbb{R}^{r}$ and piecewise smooth functions $\bar{\lambda}^{i}: I \rightarrow \mathbb{R}, \bar{\lambda}^{i} \neq 0$ for finitely many $i \in \mathbb{N}$ such that the following conditions hold:

$$
\begin{aligned}
& \sum_{i=1}^{r} \bar{\tau}^{i} f_{\bar{x}}^{i}(t)+\sum_{i \in \mathbb{N}} \bar{\lambda}^{i}(t) g_{\bar{x}}^{i}(t)=\frac{\mathrm{d}}{\mathrm{d} t}\left[\sum_{i=1}^{r} \bar{\tau}^{i} f_{\dot{\bar{x}}}^{i}(t)+\sum_{i \in \mathbb{N}} \bar{\lambda}^{i}(t) g_{\dot{\bar{x}}}^{i}(t)\right], t \in I, \\
& \int_{a}^{b} \sum_{i \in \mathbb{N}} \bar{\lambda}^{i}(t) g^{i}(t, \bar{x}, \dot{\bar{x}}) \mathrm{d} t=0, \\
& \bar{\tau} \geq 0, \sum_{i=1}^{r} \bar{\tau}^{i}=1, \bar{\lambda}^{i}(t) \geqq 0 \text { a.e., } t \in I, i \in \mathbb{N} .
\end{aligned}
$$

Proof. This theorem can be proved by using Theorem 2 and proceeding on the similar lines of ([14], Theorem 3.4).

The following example illustrates the validity of Theorem 3.

Example 4 Consider the problem (P1):

$$
\text { (P1) Minimize }\left(\int_{0}^{1}\{t-2 x(t)\}^{2} \mathrm{~d} t, \int_{0}^{1}\{2-4 \dot{x}(t)\}^{2} \mathrm{~d} t\right)
$$

Subject to

$$
\begin{aligned}
& -x(t)+\dot{x}(t) \leq \frac{k}{2}, k \in \mathbb{N}, t \in I=[0,1], \\
& x(0)=0, x(1)=\frac{1}{2} .
\end{aligned}
$$

where $x: I \rightarrow \mathbb{R}$ is a piecewise smooth state function. It is trivial that $\bar{x}(t)=\frac{t}{2}, t \in I$ is a normal efficient 
solution for (P1). It can be verified that there exist $\bar{\tau}=\left(\frac{1}{2}, \frac{1}{2}\right) \in \mathbb{R}^{2}$ and smooth functions $\bar{\lambda}^{k}: I \rightarrow \mathbb{R}, \bar{\lambda}^{k}(t)=0$, for $k \in \mathbb{N}, t \in I$ such that (27), (28) and (29) hold.

The following example illustrates that a feasible solution of (MSVP) fails to be a normal efficient solution if it does not satisfy any one of the necessary optimality conditions (27), (28) or (29).

Example 5 Consider the problem (P2):

$$
\text { (P2) Minimize }\left(\int_{0}^{1}\{x(t)+\dot{x}(t)\} \mathrm{d} t, \int_{0}^{1}\{x(t)+t\} \mathrm{d} t\right)
$$

Subject to

$$
\begin{aligned}
& x^{2}(t)+\dot{x}^{2}(t) \leq k^{2}, k \in \mathbb{N}, t \in I=[0,1], \\
& x(0)=0, x(1)=0 .
\end{aligned}
$$

where $x: I \rightarrow \mathbb{R}$ is a piecewise smooth state function. Then $\bar{x}(t)=0, t \in I$ is feasible solution for (P2). But not a normal efficient solution, since it not satisfied condition (27) for any $\left(\tau^{1}, \tau^{2}\right) \in \mathbb{R}_{+}^{2}$ and for any piecewise smooth functions $\bar{\lambda}^{i}: I \rightarrow \mathbb{R}, \bar{\lambda}^{i} \neq 0$ for finitely many $i \in \mathbb{N}, t \in I$.

\section{Topological Dual of $\left(L^{2}(I, \mathbb{R})\right)^{\mathbb{N}}$}

Let us summarizes some basic concepts and tools to find topological dual of $\left(L^{2}(I, \mathbb{R})\right)^{\mathbb{N}}$.

1) $\left(L^{2}(I, \mathbb{R})\right)^{\mathbb{N}}$ is a Riesz space ([15], p. 313) as it is partially ordered by the pointwise ordering $f \geq g$ in $\left(L^{2}(I, \mathbb{R})\right)^{\mathbb{N}}$ if and only if $f^{i} \geq g^{i}$ in $L^{2}(I, \mathbb{R})$, for each $i \in \mathbb{N}$. Its lattice operations are given pointwise

$$
\begin{aligned}
& (f \vee g)(t)=\left(\max \left\{f^{1}(t), g^{1}(t)\right\}, \max \left\{f^{2}(t), g^{2}(t)\right\}, \cdots\right) \\
& (f \wedge g)(t)=\left(\min \left\{f^{1}(t), g^{1}(t)\right\}, \min \left\{f^{2}(t), g^{2}(t)\right\}, \cdots\right) .
\end{aligned}
$$

2) $D=\left\{f=\left(f^{i}\right)_{i \in \mathbb{N}} \in\left(L^{2}(I, \mathbb{R})\right)^{\mathbb{N}} \mid f^{i} \neq 0\right.$, for finitely many $\left.i \in \mathbb{N}\right\}$ is also a Riesz space.

3) Order dual of $\left(L^{2}(I, \mathbb{R})\right)^{\mathbb{N}}$ is a Riesz space ([15], Theorem 8.24).

4) $\left(L^{2}(I, \mathbb{R})\right)$ is a Frechet lattice, as it is Banach lattice ([15], p. 348). Since countable cartesian product of Frechet lattice is Frechet lattice ([16], Theorem 5.18) which imply $\left(L^{2}(I, \mathbb{R})\right)^{\mathbb{N}}$ is Frechet lattice equipped with the product topology.

5) Given $k \in\left(L^{2}(I, \mathbb{R})\right)^{\mathbb{N}}$ define the n-tail of $k=\left(k^{i}\right)_{i \in \mathbb{N}}$ by

$$
k^{(n)}=\left(0, \cdots, 0, k^{n+1}, k^{n+2}, \cdots\right) \text {. }
$$

Motivated by the topological dual of $\mathbb{R}^{\mathbb{N}}$ ([15], Theorem 16.3), we now find the topological dual of $\left(L^{2}(I, \mathbb{R})\right)^{\mathbb{N}}$ in the following lemma.

Lemma 1 The topological dual of $\left(L^{2}(I, \mathbb{R})\right)^{\mathbb{N}}$ is

$$
D=\left\{f=\left(f^{i}\right)_{i \in \mathbb{N}} \in\left(L^{2}(I, \mathbb{R})\right)^{\mathbb{N}} \mid f^{i} \neq 0, \text { for finitely many } i \in \mathbb{N}\right\} .
$$

Proof. For any $h \in D$, define,

$$
\Psi_{h}(k)=\langle k, h\rangle=\int_{a}^{b} \sum_{i \in \mathbb{N}} k^{i}(t) h^{i}(t) \mathrm{d} t .
$$

Clearly $\Psi_{h}$ is a continuous linear functional on $\left(L^{2}(I, \mathbb{R})\right)^{\mathbb{N}}$.

For the converse, assume that $\Psi:\left(L^{2}(I, \mathbb{R})\right)^{\mathbb{N}} \rightarrow \mathbb{R}$ is continuous linear functional. The continuity of $\Psi$ at zero element of $\left(L^{2}(I, \mathbb{R})\right)^{\mathbb{N}}$ guarantees that there exist $\delta>0$ and $l>0$ such that $k \in\left(L^{2}(I, \mathbb{R})\right)^{\mathbb{N}}$ and $\left\|k^{i}\right\|<\delta$ for $i=1,2, \cdots, l$ imply $|\Psi(k)|<1$.

So for each $k \in\left(L^{2}(I, \mathbb{R})\right)^{\mathbb{N}}, n\left|\Psi\left(k^{(l)}\right)\right|=\left|\Psi\left(n k^{(l)}\right)\right|<1$ for each $n$, hence $\left|\Psi\left(k^{(l)}\right)\right|=0$. 
For each $i \in \mathbb{N}$ define $e^{i}: L^{2}(I, \mathbb{R}) \rightarrow\left(L^{2}(I, \mathbb{R})\right)^{\mathbb{N}}$ as

$$
e^{i}(f)=(0,0, \cdots, \underbrace{f}_{i \text { th place }}, \cdots, 0,0) \text {. }
$$

Then $\Psi_{o} e^{i}: L^{2}(I, \mathbb{R}) \rightarrow \mathbb{R}$ is a continuous linear functional.

By Riesz representation theorem, for $i=1,2, \cdots, l$ there exist $\lambda^{i} \in L^{2}(I, \mathbb{R})$ such that

$$
\Psi_{o} e^{i}(\alpha)=\int_{a}^{b} \alpha(t) \lambda^{i}(t) \mathrm{d} t \text {, for all } \alpha \in L^{2}(I, \mathbb{R}) .
$$

Now let $h=\left(\lambda^{1}, \lambda^{2}, \lambda^{3}, \cdots, \lambda^{l}, 0, \cdots, 0\right) \in D$,

note that $\Psi(k)=\sum_{i=1}^{l} \Psi_{o} e^{i}\left(k^{i}\right)=\sum_{i=1}^{l} \int_{a}^{b} k^{i}(t) \lambda^{i}(t) \mathrm{d} t=\langle k, h\rangle$, for each $k \in\left(L^{2}(I, \mathbb{R})\right)^{\mathbb{N}}$.

That is $\Psi=\Psi_{h}$ and $h$ is uniquely determined.

Now, if $h \geq 0$, then $\Psi_{h}(k) \geq 0$, for all $k \geq 0$

Conversely, proceeding similarly as in claim 1 of Theorem 2, it can be shown that if $\Psi_{h}(k) \geq 0$, for all $k \geq 0$ then $h \geq 0$.

This infers $h \rightarrow \Psi_{h}$ is a lattice isomorphism from $D$ onto $\left(\left(L^{2}(I, \mathbb{R})\right)^{\mathbb{N}}\right)^{\prime}$.

Hence $D=\left(\left(L^{2}(I, \mathbb{R})\right)^{\mathbb{N}}\right)^{\prime} \quad([15]$, Theorem 9.11).

\section{Conclusion}

In this paper, we have developed necessary optimality conditions for a Semi-Infinite Variational Problem. These optimality conditions are further extended to Multi-objective Semi-infinite Variational Problem (MSVP) as Theorem 3. The results proved in this article are significant for the growth of optimality and duality theory for the class of semi-infinite variational problems. An example is presented to demonstrate the validity of the theorem proved. Another example illustrates that a feasible solution of (MSVP) fails to be a normal efficient solution if it does not satisfy any one of the necessary optimality conditions stated in the theorem. Vital part of the result depends on the topological dual of $\left(L^{2}(I, \mathbb{R})\right)^{\mathbb{N}}$ which was proved as a lemma in the last section.

\section{Acknowledgements}

We thank the Editor and the referee for their comments. The first author was supported by Council of Scientific and Industrial Research, Junior Research Fellowship, India (Grant no 09/045(1350)/2014-EMR-1).

\section{References}

[1] Lopez, M. and Still, G. (2007) Semi-Infinite Programming. European Journal of Operational Research, 180, $491-518$. http://dx.doi.org/10.1016/j.ejor.2006.08.045

[2] Kanzi, N. and Nobakhtian, S. (2010) Optimality Conditions for Non-Smooth Semi-Infinite Programming. Optimization, 59, 717-727. http://dx.doi.org/10.1080/02331930802434823

[3] Mishra, S.K., Jaiswal, M. and An, L.T.H. (2012) Duality for Nonsmooth Semi-Infinite Programming Problems. Optimization Letters, 6, 261-271. http://dx.doi.org/10.1007/s11590-010-0240-8

[4] Mond, B. and Hanson, M.A. (1967) Duality for Variational Problems. Journal of Mathematical Analysis and Applications, 18, 355-364. http://dx.doi.org/10.1016/0022-247x(67)90063-7

[5] Hanson, M.A. (1964) Bounds for Functionally Convex Optimal Control Problems. Journal of Mathematical Analysis and Applications, 8, 84-89. http://dx.doi.org/10.1016/0022-247x(64)90086-1

[6] Bhatia, D. and Kumar, P. (1996) Duality for Variational Problems with B-Vex Functions. Optimization, 36, $347-360$. http://dx.doi.org/10.1080/02331939608844189

[7] Bhatia, D. and Mehra, A. (1999) Optimality Conditions and Duality for Multiobjective Variational Problems with Generalized B-Invexity. Journal of Mathematical Analysis and Applications, 234, 341-360. 
http://dx.doi.org/10.1006/jmaa.1998.6256

[8] Antczak, T. (2014) On Efficiency and Mixed Duality for a New Class of Nonconvex Multiobjective Variational Control Problems. Journal of Global optimization, 59, 757-785. http://dx.doi.org/10.1007/s10898-013-0092-8

[9] Husain, I. and Jabeen, Z. (2005) On Variational Problems Involving Higher Order Derivatives. Journal of Applied Mathematics and Computing, 17, 433-455. http://dx.doi.org/10.1007/BF02936067

[10] Luc, D.T. (1989) Theory of Vector Optimization, Lecture Notes in Economics and Mathematical Systems, Vol. 319. Springer-Verlag, New York, 75.

[11] Kreyszig, E. (1989) Introductory Functional Analysis with Applications. Wiley Classics Library, Wiley, New York, 188.

[12] Gelfand, I.M. and Fomin, S.V. (1963) Calculas of Variations. Prentice-Hall, Inc., Englewood Cliffs, 34-35.

[13] Chandra, S., Craven, B.D. and Husain, I. (1985) A Class of Non-Differentiable Continuous Programming Problems. Journal of Mathematical Analysis and Applications, 107, 122-131. http://dx.doi.org/10.1016/0022-247X(85)90357-9

[14] Mititelu, S. and Stancu-Minasian, I.M. (2009) Efficiency and Duality for Multiobjective Fractional Variational Problems with $(\rho, b)$-Quasiinvexity. Yugoslav Journal of Operations Research, 19, 85-99. http://dx.doi.org/10.2298/yjor0901085m

[15] Aliprantis, C.D. and Border, K.C. (1999) Infinite Dimensional Analysis, A Hitchhiker’s Guide. Springer-Verlag, Berlin Herdelberg, 313, 324, 348, 352, 528. http://dx.doi.org/10.1007/978-3-662-03961-8

[16] Aliprantis, C.D. and Burkinshaw, O. (2003) Locally Solid Riesz Spaces with Applications to Economics. American Mathematical Society, Providence, 127. http://dx.doi.org/10.1090/surv/105 